\title{
¿Fundamentos técnicos o tamices ideológicos? Reflexiones sobre la misión principal de las Fuerzas Armadas argentinas
}

\section{Professional underpinnings or ideological blinkers? Reflections on the primary mission of the Argentine Armed Forces}

LUCIANO ANZELINI

\section{INTRODUCCIÓN ${ }^{1}$}

En diciembre de 2015 asumió un nuevo gobierno en la Argentina. La discusión pública viene girando, desde entonces, en torno a una agenda en donde la defensa nacional detenta un lugar meramente marginal. ${ }^{2}$ Sin embargo, si se observa con detenimiento, antes de las elecciones presidenciales diversos candidatos, algunos de sus referentes más versados en la materia y académicos con inserción en el mundo de la política han planteado la necesidad de repensar el rol de los militares y su misión principal.

Se trata del resurgimiento de la pregunta que se formulara Ernesto López en los primeros años de la democracia recuperada: “¿qué hacer con los militares?” (López 1987). Este interrogante, que entonces tenía relación con la necesidad de subordinar a las fuerzas armadas al poder democrático, hoy ya no tiene el mismo cariz. Los militares argentinos se encuentran consustanciados con el régimen democrático y desde principios de la década de 1990 no han vuelto a efectuar "planteos" a los gobiernos constitucionales.

No obstante, la cuestión de la misión principal de las fuerzas armadas ${ }^{3}$ vuelve a ocupar - siempre dentro del limitado espacio de discusión sobre los asuntos castrenses - un lugar significativo como lo tenía a mediados de la década de 1980. Si bien las elucubraciones actuales, a diferencia de entonces, giran en torno de si volcar o no al instrumento militar a la lucha

Luciano Anzelini - Universidad de Buenos Aires / Universidad Nacional de Quilmes. 
contra el narcotráfico, lo que se halla en la encrucijada, al fin de cuentas, es un mismo asunto: la profesionalización de las fuerzas armadas.

Cabe recordar que, como producto de los consensos alcanzados desde la recuperación democrática en 1983, la normativa argentina establece una nítida separación entre los asuntos de defensa externa y seguridad interior (Ley de Defensa Nacional $n^{\circ}$ 23.554; de Seguridad Interior $n^{\circ}$ 24.059; y de Inteligencia Nacional $n^{\circ}$ 25.520). Este entramado normativo fue sancionado por tres gobiernos consecutivos, de diferente color político y en diferentes etapas de la Argentina contemporánea. ${ }^{4}$ Sus fundamentos constituyen los ejes vertebradores de una "política de estado" que supuso el abandono de la "doctrina de seguridad nacional" y el retorno a la "doctrina de defensa nacional” (López 2010, 254). Ello implica diferentes responsabilidades y ámbitos de actuación para los organismos castrenses y para los de seguridad. Al respecto, el decreto reglamentario de la Ley de Defensa Nacional $\left(n^{\circ} 727 / 2006\right)$ expresa:

[...] deben rechazarse enfáticamente todas aquellas concepciones que procuran extender y/o ampliar la utilización del instrumento militar hacia funciones totalmente ajenas a la defensa, usualmente conocidas bajo la denominación 'nuevas amenazas', responsabilidad de otras agencias del Estado organizadas y preparadas a tal efecto.

El presente artículo parte de una premisa básica: aquellos que, alegando una sustancial modificación del contexto político actual respecto del de la década de 1980, plantean la necesidad de una modificación de la misión principal de las fuerzas armadas desatienden un conjunto de aspectos políticos, técnicos y militares que hacen al núcleo duro de la profesión castrense. El asunto se torna doblemente problemático cuando se atribuye el supuesto anacronismo de la actual misión principal de las Fuerzas Armadas a razones de orden político-ideológico, pues al hacerlo pierden de vista las enseñanzas estratégicas y operacionales que legó la Guerra de Malvinas (1982) a la Argentina democrática. Dicha omisión supone la inadecuada internalización de la única experiencia concreta de un país sudamericano que enfrentó a una gran potencia en una guerra bilateral a lo largo del siglo XX.

Adicionalmente, se parte de una segunda premisa: no son prejuicios ideológicos los que se hallan en la base de una postura refractaria al empleo de las fuerzas armadas en la lucha contra el narcotráfico. Son, antes bien, argumentos técnico-militares los que desaconsejan hacer del tráfico de drogas un asunto de incumbencia prioritaria de las fuerzas armadas.

El trabajo se organiza del siguiente modo: en la siguiente sección se efectúa una breve descripción de las perspectivas, tanto políticas como académicas, que plantean la necesidad de revisar la misión principal de las 
fuerzas armadas. En el tercer apartado se polemiza con dichas posiciones y se enumera un conjunto de fundamentos estratégicos y técnicos que reafirman el marco normativo vigente. Asimismo, se identifica el conjunto de argumentos que configuran el actual clima de opinión favorable a la militarización de la seguridad pública. Finalmente, se extraen una serie de conclusiones, basadas en el desempeño de las fuerzas armadas argentinas durante el siglo XX, relativas a los efectos que podría acarrear en términos de desprofesionalización el vuelco del instrumento militar a la lucha contra el narcotráfico.

\section{VOCES EN CAMPAÑA Y MEDIDAS EN FAVOR DE LA MILITARIZACIÓN DE LA SEGURIDAD PÚBLICA}

El candidato presidencial del Frente para la Victoria (FPV), Daniel Scioli, planteó que habría que "evaluar" el rol de las Fuerzas Armadas en la lucha contra el narcotráfico: "Como mi compromiso es la lucha contra la inseguridad y el narcotráfico es una realidad, vamos a implementar un blindaje de nuestros 2.500 kilómetros de fronteras. Lo haremos con efectivos de las fuerzas de seguridad y Fuerzas Armadas que ya están allí actuando en los escudos Norte y Noroeste" (La Nación 2014). ${ }^{5}$

Más directo todavía fue Sergio Massa, candidato de la alianza Unidos por una Nueva Alternativa (UNA). En Rosario (provincia de Santa Fe), una de las ciudades más afectadas por el narcotráfico, afirmó:

Es muy importante que cada rosarino sepa qué vamos a hacer en materia de lucha contra la inseguridad y el narcotráfico. Vamos a llevar a las Fuerzas Armadas a los barrios que hoy están copados por los narcos. Vamos a usar las fuerzas armadas para detener nuestra frontera en materia de blindaje contra el narcotráfico. Vamos a tener ley de derribo (Infobae 2015).

Por su parte, el actual presidente Mauricio Macri, pese a haber sido ambiguo durante la campaña electoral, desde su asunción ha avanzado en dirección a la militarización de la seguridad pública. A menos de un mes de haber asumido, emitió el Decreto n 228/2016, que declaró la "Emergencia de Seguridad Pública" en todo el territorio nacional por el lapso de un año, "con el objeto de revertir la situación de peligro colectivo creada por el delito complejo y el crimen organizado". La norma autoriza al instrumento militar, en el marco de sus actividades de control del espacio aéreo, a utilizar fuerza letal contra aviones que no respondan a las advertencias efectuadas. El decreto puso en vigencia, con vistas a la conjuración de problemáticas del campo de la seguridad pública, un conjunto de "reglas de 
protección aeroespacial” cuyas autoridades de aplicación y ejecución son funcionarios de las Fuerzas Armadas.

Al margen de la discusión respecto de la eficacia histórica de este tipo de medidas, y de que su implementación podría conllevar una situación de pena de muerte sin juicio previo (Ugarte 2009; Garré 2013), lo que está fuera de discusión es que el decreto contradice el plexo normativo. Según León Arslanián: "el tema del derribo viola por empezar dos leyes: la de Seguridad Interior y la de Defensa Nacional, que prohíben la actuación de las fuerzas militares o armadas en esta materia. De modo que utilizar la Fuerza Aérea con esa finalidad tiene un problema serio de carácter legal” (Tres Líneas 2016). La Ley de Seguridad Interior no 24.059 prevé la participación del instrumento militar en cuestiones de seguridad pública solamente para tres casos y de manera excepcionalísima. ${ }^{6}$ Lo instruido en el decreto de "Emergencia de Seguridad Pública” por el presidente Macri no se ajusta a lo contemplado en ninguno de esos casos.

Por otra parte, tras la visita a la Argentina en marzo de 2016 del entonces presidente de los Estados Unidos, Barack Obama, Buenos Aires y Washington sellaron un conjunto de acuerdos en materia de cooperación en seguridad y defensa. Sin distinguir entre las responsabilidades de los organismos militares y los de seguridad, el espectro de cuestiones convenidas incluye la lucha contra el narcotráfico y el terrorismo, la asistencia en la Triple Frontera, el establecimiento de centros de fusión de inteligencia y cuestiones inherentes a la defensa hemisférica y a las fuerzas de seguridad en el Comando Sur (Verbitsky 2016). Los acuerdos suponen la aceptación por parte de la Argentina de un conjunto de lineamientos estratégicos contenidos en los documentos del Pentágono para nuestra región. Allí Washington impulsa que los países latinoamericanos empleen a sus instrumentos militares para enfrentar los "desafíos multifacéticos de la seguridad” (Departamento de Defensa 2012). ${ }^{7}$

También en el plano de las declaraciones el gobierno de Mauricio Macri instaló la cuestión de la militarización de la seguridad pública. El ex ministro de Defensa, Julio Martínez, luego de reunirse en abril de 2016 con Rose Gottemoeller, subsecretaria de los Estados Unidos para el Control de Armas y Asuntos de Seguridad Internacional, afirmó: "Avanzamos en una serie de temas que incluye [...] la lucha contra el narcotráfico" (Ministerio de Defensa 2016a). Tres meses después, el entonces ministro se reunió con el comandante en Jefe de la Fuerza Terrestre de la Federación Rusa, coronel general Oleg Salyukov, con quien dialogó "sobre la necesidad de [...] intercambiar conocimientos en temas relacionados con la lucha contra el terrorismo" (Ministerio de Defensa 2016b). Resulta difícil explicar los avances e intercambios que menciona Martínez, excepto que efectiva- 
mente se trate de una violación a las responsabilidades que la normativa le confiere al área de la Defensa Nacional.

La más importante de las manifestaciones relativas al empleo de las Fuerzas Armadas en la conjuración de amenazas de la seguridad pública ha surgido del propio presidente Macri. Durante la celebración del Día del Ejército Argentino el 30 de mayo de 2016 expresó: "Necesitamos que las Fuerzas Armadas se adapten a las demandas y requerimientos del siglo XXI $[\ldots]$ necesitamos que trabajemos juntos contra las amenazas del terrorismo” (Télam 2016).

\section{La militarización de la seguridad pública en la perspectiva académica}

Dentro del campo académico resultan interesantes las miradas de Ángel Tello y Julio Spota, ${ }^{8}$ quienes han reflexionado sobre algunas de las cuestiones que estructuran el presente trabajo (Tello; Spota 2015). Los autores consideran anacrónicas a las leyes vigentes y enfatizan las razones políticas que, a su juicio, estarían obturando una necesaria revisión del plexo normativo. El argumento que esgrimen es que este último resulta inadecuado a los tiempos que corren y que los motivos que justifican su sostenimiento son de carácter ideológico. Asimismo, plantean que la razón que justificó la división de responsabilidades entre los organismos militares y los de seguridad pública al momento de la sanción de la Ley de Defensa Nacional (1988) ya no opera en la actualidad:

Acaso el mayor desafío en la República Argentina actual surja en la superación de principios ideológicos operantes en la forma de tamices cognoscitivos [...] el empleo de las Fuerzas Armadas (FFAA) está reservado exclusivamente a repeler y neutralizar acciones armadas propiciadas por unidades estatales. La lógica originaria de la restricción impuesta a la praxis castrense reside en la coyuntura histórico-política atravesada por la República Argentina en la década de 1980 [...] Si bien la Ley de Defensa Nacional data de 1988, fecha que explica su espíritu, la reglamentación fue implementada recién en 2006, momento en que la tesitura nacional en términos de institucionalidad con arreglo a la Constitución cancelaba la posibilidad de asomo alguno de levantamiento armado [...] lo que en 1988 resultaba lógico en términos coyunturales fue enfatizado en 2006 en apelación a un análisis anacrónico de la realidad argentina en materia de relaciones cívico-militares" (Tello; Spota 2015, 24-26).

Los autores refieren a la reglamentación de la Ley de Defensa Nacional como el momento paradigmático de un supuesto involucramiento subjetivista del legislador, que inhibiría un abordaje estratégico acorde a los 
desafíos del siglo XXI. Plasmada luego de 18 años de postergación, la reglamentación a la que aluden Tello y Spota se ha erigido en el "nudo gordiano" a desatar por parte de los sectores que impulsan el vuelco de las Fuerzas Armadas a la lucha contra el narcotráfico:

La limitada viabilidad práctica derivada de las restricciones operativas impuestas por la reglamentación en vigencia, instituyen un rango de aplicación artificialmente limitado de cara al formato de los retos contemporáneos. Frente a la multidimensionalidad e imbricación de las amenazas cernidas en la actualidad y el porvenir, continuar manteniendo el esquema de seguridad interior y defensa nacional como esferas radicalmente diferenciadas entre sí supone un intento ímprobo de forzar la realidad dentro de la teoría (Tello; Spota 2015,33).

Sucede que la naturaleza híbrida de las nuevas amenazas demanda soluciones creativas y actualizadas al formato específico presentado por el desafío estratégico que representan $[\ldots]$. La vastedad del problema erigido por las 'nuevas amenazas' exige la construcción de una solución integral donde ingresen en igual medida la corrección de los errores actuales y una revisión general de reglamentación de la Ley de Defensa Nacional, junto a la formulación de esquemas estratégicos nacionales capaces de lidiar con las amenazas reales cifradas en el horizonte de posibilidades abierto para la República Argentina en el siglo XXI (Tello; Spota 2015, 33-34).

Tanto las expresiones políticas reseñadas como los argumentos académicos enumerados omiten la mención a aspectos técnico-profesionales, que constituyen el eje vertebrador del andamiaje jurídico vigente. En el caso de Tello y Spota, al ceñirse al plano político-ideológico de la década de 1980 como variable justificadora del entramado normativo vigente, desatienden una multiplicidad de factores técnico-militares que convendría no perder de vista. En particular, como se precisará en la próxima sección a partir de la revisión del "Informe Rattenbach", ${ }^{9}$ aquellos relativos al fracaso estratégico y operacional de la Guerra de Malvinas (1982).

Por otra parte, cabe advertir que existen tres argumentos sobre los que se apoyan quienes se inclinan por la militarización de la seguridad pública en la Argentina:

a) que las Fuerzas Armadas representan, en las actuales condiciones, una estructura ociosa carente de misión y que, por ende, se les debe asignar nuevos desafíos; 
b) que el carácter improbable de nuevos enfrentamientos bélicos convencionales con otros estados fortalece el argumento de la carencia de misión y de la ociosidad de las estructuras militares; y

c) que el actual esquema normativo se sustenta en una tesis anacrónica: la de las Fuerzas Armadas como actores centrales del sistema político.

Los argumentos que a continuación se enumeran buscan exhibir que, en realidad, los "tamices cognoscitivos" podrían estar operando del lado de quienes buscan desandar una verdadera política de Estado de la democracia argentina. Tras el reclamo de "soluciones creativas" para hacer frente a un entorno estratégico caracterizado por la "naturaleza híbrida de las nuevas amenazas", se detectan al menos tres cuestiones: en primer lugar, la adopción de una postura que reproduce, desde el nivel político, los intereses corporativos de determinados sectores castrenses "en busca de misión" (Scheetz 2011,78); segundo, una apreciación incorrecta del estadio actual de evolución de las problemáticas criminales complejas (en particular del narcotráfico); y en tercer lugar, una llamativa omisión de las enseñanzas estratégicas y operacionales de la guerra del Atlántico Sur, que exhibieron cuán caro resultó la desprofesionalización castrense tras décadas de adiestramiento, adoctrinamiento y formación alejados de su misión principal.

\section{A FAVOR DE UNA CONCEPCIÓN PROFESIONALIZADA DE LAS FUERZAS ARMADAS}

Desde la recuperación de la democracia, la Argentina ha representado - desde el punto de vista normativo - una versión "típico-ideal" de distinción de responsabilidades entre las fuerzas militares y las de seguridad. Por el contrario, en la mayor parte de América Latina los organismos castrenses han asumido crecientes atribuciones en materia de seguridad pública (Pion-Berlin 2008). ${ }^{10}$ Las otras excepciones que acompañan a la Argentina en un abordaje semejante del uso de la fuerza militar son Chile y Uruguay (Cels 2015, 374-375).

El revival ${ }^{11}$ de declaraciones de funcionarios argentinos en favor de la militarización de la seguridad interior y las primeras materializaciones de esta inclinación (Decreto de Emergencia de Seguridad Pública) conducen a reflexionar sobre un tópico introductorio de los cursos de Defensa Nacional: aquel relativo a las definiciones de defensa vis a vis seguridad.

En efecto, los militares difieren de los policías en cuanto a sus objetivos de intervención y, por ende, en lo que hace a sus medios, formación, doctrina, inteligencia y adiestramiento en el uso de la fuerza pública. Los militares se forman para "dar la vida por la patria” en una guerra (Salvi 
2013), no para enfrentar redes de narcotraficantes. Las estructuras castrenses, a diferencia de los policías, no están preparadas para el uso gradual de la fuerza, ya que el principio de proporcionalidad no está presente en su adiestramiento. Sus intervenciones, por lo tanto, tienen un mayor grado de letalidad (Saint-Pierre 2008, 18-19). ${ }^{12}$

El paulatino desvanecimiento de esta natural división de responsabilidades entre organismos castrenses y de seguridad - proceso que ya se ha extendido en América Latina en términos generales y que desde hace un tiempo se proyecta sobre la Argentina con renovada fuerza - se ve traccionado por una serie de circunstancias.

En primer lugar, la existencia de presiones internacionales sostenidas desde el fin de la Guerra Fría, en las que Washington detenta un papel significativo (Withers, Santos e Isacson 2010). La desaparición de los "enemigos ideológicos internos", eje estructurante de la "Doctrina de Seguridad Nacional”, condujo en América Latina a un replanteo de las misiones castrenses. Ello, sumado a la generalizada distensión de las relaciones interestatales como consecuencia de la disolución de las viejas hipótesis de conflicto, aceleró la transición hacia las "nuevas amenazas" como hipótesis de empleo de los organismos militares (López y Sain 2004). De este modo, las presiones de Washington - acompañadas de cuantiosos recursos y posibilidades de capacitación - operaron efectivamente para que muchos presidentes latinoamericanos, durante las últimas décadas, asignaran misiones no idóneas al personal militar, en primer lugar la lucha contra el narcotráfico. Se materializa así una paradoja: los Estados Unidos persuaden a otros países para que sus hombres de armas asuman misiones no convencionales, algo que los militares norteamericanos no realizan en el interior de su propio territorio (Verbitsky 2011).

No debe perderse de vista que, tras décadas de auge del enfoque de la "guerra contra las drogas" - que ubica en el centro a la militarización de la seguridad pública -, los países latinoamericanos más consustanciados con esta concepción estratégica (Colombia y México) han comenzado a replantear sus posturas. ${ }^{13} \mathrm{El}$ aumento de los niveles de violencia en los conflictos internos, las violaciones a los derechos humanos y el incremento de hechos de corrupción por parte de las fuerzas armadas resultan signos evidentes de agotamiento de este curso de acción (CELS 2015, 371).

En segundo término, cabe señalar las expresiones de una dirigencia política no formada técnicamente en los asuntos de seguridad y defensa, pero sí ávida de dar respuestas perentorias a una ciudadanía aquejada por el avance del narcotráfico. Como señala Juan Tokatlian:

[...] las manifestaciones de Scioli, Macri, Michetti, De Narvaéz y Massa a favor de políticas de 'mano dura' con un rol activo de las 
fuerzas armadas para frenar ese fenómeno, constituyen ejemplos emblemáticos del recurso a los lugares comunes (Tokatlian 2014).

Esta apelación al lugar común de la "mano dura" encuentra un campo fértil en una ciudadanía que, ante la falta de soluciones inmediatas, refuerza la demagogia punitivista de sus dirigentes. Así, los reclamos de endurecimiento de las respuestas penales se solapan con las propuestas de utilizar a los militares en la lucha contra el narcotráfico, sin un diagnóstico claro acerca de la efectividad que podrían acarrear estas medidas. A esta peligrosa confluencia punitivista se suma la caracterización - que expresa una parte significativa de la dirigencia y de la opinión pública - de las Fuerzas Armadas como estructuras ociosas carentes de misión operativa.

Un tercer aspecto de este panorama es la perspectiva de los propios militares argentinos. Puesto simplemente: ¿cuál es la mirada de los hombres de armas en relación con su potencial empeñamiento en la lucha contra el tráfico de drogas? Sobre la autopercepción castrense se conjugan: por un lado, los factores ya mencionados, esto es, las presiones internacionales (particularmente de los Estados Unidos), una dirigencia política poco esclarecida en estos asuntos, y la demagogia punitivista tanto de los dirigentes como de la ciudadanía; y por el otro, la tendencia de la propia organización burocrático-militar a una "búsqueda de misión" en ausencia de directrices políticas claras.

Esto significa que uno de los elementos a ponderar es el posicionamiento de las propias Fuerzas Armadas. En efecto, la participación castrense en asuntos de seguridad interior podría representar para las organizaciones militares el acceso a recursos - medios materiales y formación - no accesibles de otro modo (Olson; Haugaard; Isacson 2007). Asimismo, podría implicar, por la vía de la expansión de roles, un fortalecimiento de las estructuras castrenses como actores de peso en la escena política.

La lenta proyección de las fuerzas armadas sobre asuntos de la agenda no ligados a su misión principal no es algo imprevisto para el campo de las políticas públicas. Es natural que ante una dirigencia incapaz de instalar una discusión seria acerca de la profesionalización militar - y por ende "corta de miras" para establecer directrices político-estratégicas claras sobre su misión -, los hombres de armas emprendan autónomamente una búsqueda de responsabilidades. El problema mayor no es en este caso la tendencia al "autodiseño" de una organización que experimenta una crisis de identidad, sino la defección de la clase política a la hora de conducir estratégicamente al instrumento militar (Sain 2001, 45; Scheetz 2011, 78).

Un cuarto elemento - llamativamente el más olvidado - es la experiencia de la Guerra de Malvinas en 1982. La Argentina fue el único estado 
latinoamericano que durante el siglo XX enfrentó a una gran potencia (Gran Bretaña) en una guerra convencional de carácter bilateral. El conflicto del Atlántico Sur exhibió hasta qué punto la desprofesionalización castrense $^{14}$ resultante de la politización de los organismos militares (López 2007, 19), sumado a la desnaturalización del rol principal de las fuerzas armadas como consecuencia de la denominada "guerra antisubversiva", condujo a un rotundo fracaso en lo que hace al núcleo duro de la actividad militar. Por lo tanto, interpretar la vigencia del actual entramado jurídico como resultado de la acción de una dirigencia política atada a supuestas anteojeras ideológicas, o bien como la secuela de una apreciación estratégica demodé incapaz de captar las transformaciones del escenario de seguridad global, expresa una llamativa desatención de las enseñanzas de la guerra, quintaesencia de la profesión militar.

Como lo refleja el "Informe Rattenbach", la sumatoria de errores cometidos en los planos estratégico y operacional en Malvinas expresa a todas luces el resultado esperable en un conflicto convencional cuando los responsables de la conducción pierden de vista la preparación para su misión principal. El instrumento militar es una estructura burocrático-profesional que se adiestra para la eventualidad de una guerra en tiempos de paz. Cualquier misión subsidiaria ${ }^{15}$ - por ejemplo, la contribución al sistema de seguridad interior en excepcionalísimas circunstancias - debe ser atendida con capacidades remanentes o de uso dual, y no debe atentar contra la misión primaria y esencial que es la preparación para la guerra (Montenegro 2008, 15-16). El impacto que tuvo la desprofesionalización castrense a lo largo del siglo XX como resultado de la politización y el adiestramiento contrainsurgente (García; Ballester; Rattenbach; Gazcón 1987, 48-49) podría replicarse en el siglo XXI con un instrumento militar volcado a tareas de investigación y represión policial. Las "nuevas amenazas”, en primer lugar el narcotráfico, podrían fungir como vehículos de una renovada desprofesionalización.

La derrota de Malvinas, según pone en evidencia el "Informe Rattenbach”, expresa algunas de las consecuencias de la desnaturalización que experimentan las fuerzas armadas cuando, por motivos políticos, son alejadas de la adecuada preparación para su misión principal: la defensa en una guerra interestatal.

De las conclusiones a las que arribó la comisión presidida por el Teniente General Benjamín Rattenbach, es posible extraer algunas enseñanzas que hacen a la dimensión estrictamente profesional de los hombres de armas. En particular, cabe puntualizar las siguientes falencias de orden estratégico nacional y militar: a) el quebrantamiento de las más elementales normas de Planificación de la guerra según lo estipulado en el Sistema 
Nacional de Planeamiento, lo que conllevó, entre otros sucesos, el adelantamiento indebido de la "Operación Rosario" (anteriormente "Operación Azul") destinada a retomar el control de la capital de las Islas Malvinas; b) la inexistencia de un plan concreto de defensa de las islas del Atlántico Sur para el caso de que Gran Bretaña intentase recuperarlas por medio de una "acción bélica de magnitud"; c) la falta de preparación en materia de inteligencia estratégica, tanto nacional como militar, lo que implicó, entre otras cuestiones, una falta de conocimiento sobre los ambientes estratégicos de interés con impacto negativo en el empleo de los medios empeñados; d) la deficiente preparación desde el punto de vista logístico, área especialmente sensible a las improvisaciones; y e) la carencia del adecuado adiestramiento para la acción conjunta, lo que se tradujo en la ausencia de unidad de comando y en el desarrollo de operaciones sin coherencia, integración y convergencia de esfuerzos entre las tres Fuerzas Armadas (Comisión de Análisis y Evaluación de las Responsabilidades Políticas y Estratégico Militares en el Conflicto del Atlántico Sur 2012 [1982], 41, 217, 218, 222, 233, 238, 239).

En síntesis, el "Informe Rattenbach" constituye un material imprescindible tanto para los decisores de políticas públicas como para los analistas de los temas castrenses. Pese a la valentía de los soldados que combatieron en el teatro de operaciones y al eficiente desempeño de muchas de las unidades desplegadas, el conflicto del Atlántico Sur puso blanco sobre negro el destino inevitable de unas Fuerzas Armadas que habían atravesado medio siglo de politización y “policialización” (Salazar 2009). La siguiente advertencia de la Comisión que redactó el Informe resulta esclarecedora:

Al realizar el análisis y evaluación de lo actuado en el Conflicto del Atlántico Sur, esta Comisión ha advertido y comprobado una serie de circunstancias y hechos que constituyen importantes experiencias de naturaleza política y militar. Una vez elaboradas, podrán proporcionar valiosas enseñanzas, las que contribuirán a perfeccionar la organización integral de la Nación para el logro de sus objetivos políticos en situaciones de conflicto, actualizarán nuestra doctrina para el empleo del potencial de guerra y del poder militar, mejorarán los conocimientos y procedimientos de empleo de medios modernos en el combate y acrecentarán la aptitud profesional de los mandos, como así también de las tropas de nuestras FFAA [...]. Este ha sido el único conflicto bélico de la Nación en lo que va del siglo. Si bien la paz es un objetivo nacional permanente de cualquier comunidad civilizada, ella se logra mediante una adecuada preparación para disuadir a los enemigos potenciales de emplear la fuerza en contra propia $(2012,284,287)$. 
Un quinto elemento que no puede ser obviado es la inadecuación de las Fuerzas Armadas como instrumento para combatir al narcotráfico en su estadio actual de desarrollo (Sain 2001; Garré 2015). Aún cuando no existe ningún diagnóstico serio producido por las autoridades nacionales acerca de la evolución de esta problemática criminal compleja, la sofisticación de estos emprendimientos resulta aún precaria - si bien creciente - en la Argentina. Las organizaciones dedicadas al tráfico de drogas no emplean en la fase actual sistemas de armas mayores ni tácticas militares. ${ }^{16} \mathrm{Y}$ aún en el hipotético caso de que esto sucediera en el mediano o largo plazo, la Argentina contaría para lidiar con ello con fuerzas intermedias o militarizadas, como son la Gendarmería Nacional (GNA) o la Prefectura Naval (PNA).

En las presentes condiciones se requiere, ante todo, expertise en materia de inteligencia criminal e investigación compleja para desarticular a las bandas que operan en el territorio nacional. Cualquier análisis perspicaz de la cuestión arrojará, por ejemplo, que lavado de activos y narcotráfico marchan en tándem. Por este motivo, es difícil ponderar cuál sería la efectividad de emplear el máximo poder de fuego del estado en una materia que, en la situación actual, requiere ante todo de capacidad investigativa.

Asimismo, resulta indispensable contar con un diagnóstico certero sobre la evolución del narcotráfico fronteras adentro; así como tener una mirada esclarecida sobre el lugar de la Argentina en el "mercado mundial" de drogas. Advertir la especificidad de la realidad local, esto es, el modo en que se estructuran los "mercados internos", es un paso indispensable para no terminar aplicando estrategias diseñadas en otras latitudes que han demostrado ser ineficaces en casi todos los países de la región. ${ }^{17}$

Por otra parte, los cuerpos policiales requieren de una preparación adecuada para actuar como auxiliares de la justicia, lo que implica la adquisición de capacidades imprescindibles en materia de colaboración con juzgados y ministerios públicos fiscales. Cualquier lectura atenta de los programas de estudio de los oficiales de las Fuerzas Armadas argentinas en las tres etapas del plan de carrera que componen la academia militar, la escuela de guerra específica y la escuela de guerra conjunta - revela la ausencia de materias relativas a estas competencias. En consecuencia, existe una desvinculación entre los programas de estudios de quienes se forman para ser profesionales de la guerra y los saberes específicos requeridos por los expertos en la lucha contra la criminalidad compleja.

Más que pensar en el involucramiento de las Fuerzas Armadas en la lucha contra el narcotráfico, la actual dirigencia podría analizar alguna de las propuestas más innovadoras que plantean los expertos. En particular, aquella consistente en establecer una Agencia Federal de Lucha contra 
el Delito Complejo (Sain 2013). En este sentido, debería profundizarse la discusión pública acerca de las ventajas de estructurar una agencia de este tipo a nivel nacional que, siguiendo los modelos más avanzados a nivel mundial, refuerce la capacidad del Estado de desarrollar labores de inteligencia e investigación criminal a partir de las capacidades existentes en las fuerzas de seguridad.

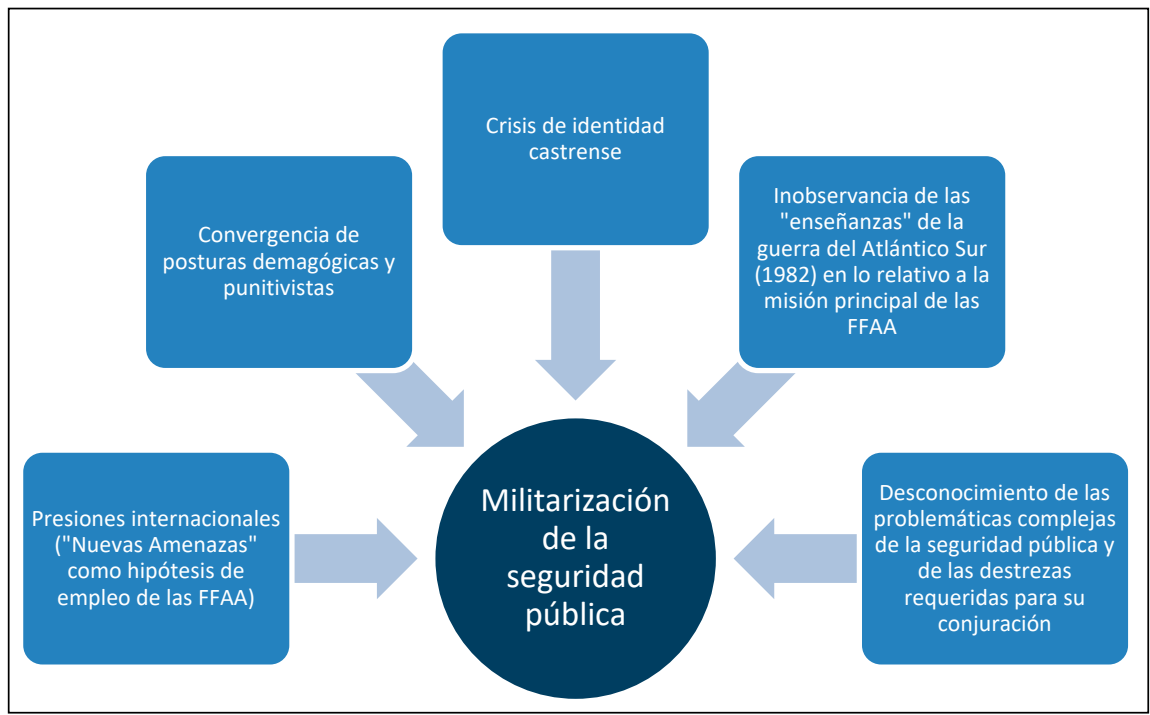

Figura 1 - Factores contribuyentes a la "militarización de la seguridad pública" en la Argentina.

Fuente: elaboración del autor.

\section{CONCLUSIONES}

La misión principal de las Fuerzas Armadas se halla nuevamente en discusión en la Argentina. Esto sucede en un contexto en el que se han desmantelado las hipótesis de conflicto con los países vecinos y se ha expandido en la región una dinámica cooperativa en materia de seguridad internacional. A su vez, las principales problemáticas de seguridad que aquejan a los países latinoamericanos tienen que ver con el universo de las denominadas "nuevas amenazas", en particular el narcotráfico.

En consecuencia, el marco en el que se desenvuelven las Fuerzas Armadas se halla reconfigurado. El peso de los asuntos ligados a la preservación de la soberanía territorial y a la protección de la nación frente a 
eventuales agresiones predatorias de otros estados resulta sustantivamente menor que en otros momentos de la historia argentina. Por lo tanto, con bastante sensatez, el lector podría formularse la siguiente pregunta: ¿para qué sostener en este escenario a estructuras costosas como son las Fuerzas Armadas? Y a renglón seguido, se podría adicionar un segundo interrogante: ¿por qué no volcarlas, como hace la mayoría de los países latinoamericanos, a la lucha contra el narcotráfico y otras problemáticas de la seguridad pública que encabezan las preocupaciones ciudadanas?

La respuesta a estas cuestiones debe partir de dos premisas: por un lado, que la realidad internacional, por más cooperativa que pueda parecer, continúa reposando en un sistema basado en la capacidad de autodefensa de los Estados. En este sentido, la persistencia de conflictos irresueltos aún en "regiones de paz", sumada a la existencia de grandes espacios territoriales potencialmente provechosos en materia de recursos naturales, amerita la preservación de un instrumento militar en condiciones de efectuar, como última ratio y a través del máximo poder de fuego del Estado, la legítima defensa de los intereses de la nación.

Por otra parte, la postura sostenida en este ensayo es que el nuevo escenario de seguridad global y regional no ofrece razones suficientes para justificar una alteración de la misión principal de las Fuerzas Armadas argentinas (Montenegro 2008, 19). Es aquí donde emerge un argumento fundamental. Que hoy no se visualice, de manera evidente, un potencial enemigo militar externo, y que a la vez las principales problemáticas que se ciernen sobre los países latinoamericanos provengan de las "nuevas amenazas”, no es una razón atendible para volcar a las fuerzas armadas a tareas de naturaleza policial.

La asunción de Mauricio Macri, en diciembre de 2015, ha significado un punto de inflexión. Más allá del lugar marginal de los asuntos castrenses en la agenda de gobierno, una medida puntual - la sanción del Decreto $\mathrm{n}^{\circ}$ 228/2016 que estableció la "Emergencia de Seguridad Pública" y habilitó el derribo por la fuerza militar de aeronaves civiles - ha despertado numerosas críticas. La decisión ha sido juzgada como un avasallamiento sobre el ordenamiento normativo vigente en materia de defensa externa y seguridad interior. A esta decisión concreta se han sumado las declaraciones de funcionarios públicos, incluidas las del propio presidente y de su ministro de Defensa, abogando por la intervención de las fuerzas armadas en la lucha contra el narcotráfico y el terrorismo.

Estas posiciones no son sustentadas solamente por funcionarios del actual gobierno, sino que empiezan a cobrar fuerza en diferentes sectores políticos y sociales. Como se señaló, los principales contendientes electorales del actual presidente, Daniel Scioli y Sergio Massa, fueron durante 
la campaña más explícitos todavía que el actual mandatario en cuanto a la potencial participación de las fuerzas armadas en la lucha contra el narcotráfico. También sectores académicos han planteado la necesidad de revisar el entramado jurídico forjado a lo largo de tres décadas de democracia, con el objeto de hacer frente "más creativamente" a la "naturaleza híbrida de las nuevas amenazas".

El argumento central del artículo es que este tipo de postura pierde de vista muy importantes argumentos técnico-profesionales, al margen de muy atendibles criterios políticos. A la hora de enumerar estos elementos, se ha procedido a enfatizar los de orden profesional, con el objeto de poner en entredicho las afirmaciones que plantean que el rechazo al involucramiento de las Fuerzas Armadas en cuestiones de seguridad interior surge de posiciones de carácter político-ideológico.

Quienes postulan el desembarco del instrumento militar en la lucha contra las "nuevas amenazas" suelen señalar como inevitable tal decisión, entre otras razones, por las siguientes: i) las fuerzas armadas son en la actualidad estructuras carentes de una misión operativa fácilmente identificable; ii) la "zona de paz regional" fortalece el argumento de la carencia de misión y de la ociosidad de las estructuras castrenses; y iii) las fuerzas armadas ya no son un actor pretoriano del sistema político.

Frente a esta perspectiva, la mirada de este ensayo procura seguir pensando al instrumento militar desde una concepción profesionalizada, centrada en su misión principal. Las posturas que ubican a las "nuevas amenazas" como hipótesis de empleo del instrumento militar en la Argentina, detentan tres problemas básicos: a) reproducen los intereses corporativos de unas Fuerzas Armadas "en busca de misión”; b) desconocen el estadio actual de evolución de las problemáticas criminales complejas y los saberes técnico-profesionales requeridos para su conjuración; y c) omiten una serie de enseñanzas históricas fundamentales en términos estratégicos relativos a la misión principal de las fuerzas armadas, paradojalmente en el único estado latinoamericano que libró una guerra convencional contra una gran potencia en el siglo XX.

Asimismo, el artículo identifica cinco elementos específicos que contribuyen a forjar un clima político-social contribuyente a la militarización de la seguridad pública. En primer lugar, las presiones internacionales - particularmente de los Estados Unidos - para hacer de las "nuevas amenazas" una hipótesis de empleo de las Fuerzas Armadas, en una línea similar a lo acontecido en casi toda América Latina, luego del fin de la Guerra Fría.

En segundo lugar, la combinación de una dirigencia política no esclarecida en el manejo de los asuntos castrenses y una ciudadanía visceralmente 
atravesada por el fenómeno del narcotráfico. Esta convergencia fortalece las posturas demagógico-punitivistas de los líderes ante el reclamo de endurecimiento de las penas por parte de los ciudadanos. El resultado es que ambos sectores propician como salida el recurso a los militares para frenar el narcotráfico.

En tercer lugar, la crisis de identidad castrense frente al contexto reseñado. Con ausencia de directrices políticas claras, presupuestos escuálidos, presiones internacionales y una organización burocrático-militar con "instinto de supervivencia", se torna factible la aceptación por parte de las cúpulas castrenses de nuevos desafíos de naturaleza no militar propios del campo de la seguridad pública. Esta situación además les permitiría a los hombres de armas recuperar cierta capacidad de interlocución política.

En cuarto término, la llamativa inobservancia de las enseñanzas estratégico-nacionales, estratégico-militares, estratégico-operacionales y táctico-operacionales de la guerra del Atlántico Sur (1982), que ponen de manifiesto el resultado esperable en una contienda convencional, cuando las Fuerzas Armadas se alejan sistemáticamente de la preparación para la guerra. El artículo plantea que este camino de desprofesionalización resultado de la politización y "policialización” alcanzadas durante el siglo XX - podría replicarse en el siglo XXI como resultado de una reorientación hacia a las "nuevas amenazas".

Finalmente, cabe señalar la omisión de aspectos profesionales del campo de la seguridad pública que advierten acerca de la ineficacia de los cuerpos militares para hacer frente a problemáticas criminales, más aún en un país que cuenta con fuerzas intermedias o militarizadas bien equipadas y con saberes específicos para hacer frente a estos desafíos. Una organización compleja dedicada al narcotráfico o al terrorismo debe ser neutralizada a través de cuerpos de prevención, inteligencia criminal e investigación, que poco o nada tienen que ver con la preparación de los profesionales para la guerra.

En resumidas cuentas, existen muy razonables fundamentos político-técnicos (no tamices ideológicos) para continuar respaldando una concepción profesionalizada de la Defensa Nacional, con unas fuerzas armadas adecuadamente preparadas para su misión principal.

\section{REFERENCIAS}

Centro de Estudios Legales y Sociales (CELS). 2015. Derechos humanos en Argentina. Informe 2015. Buenos Aires: Siglo Veintiuno Editores. 
Comisión de Análisis y Evaluación de las Responsabilidades Políticas y Estratégico Militares en el Conflicto del Atlántico Sur. 2012 [1982]. Informe final. Buenos Aires: Poder Ejecutivo Nacional.

Departamento de Defensa de los Estados Unidos. 2012. La política de Defensa para el Hemisferio Occidental. Recuperado de: http://archive.defense.gov/news/ WHDPS-Spanish.pdf [21 de julio de 2016].

García, J. L., Ballester, H., Rattenbach, A., Gazcón, C. 1987. Fuerzas Armadas argentinas el cambio necesario. Bases políticas y técnicas para una reforma militar. Buenos Aires: Editorial Galerna.

Garré, N. 2013. Una ley de derribo es inconstitucional. Página 12. 18 de noviembre.

Garré, N. 2015. El narcotráfico y los militares. Página 12. 12 de octubre.

Infobae. 2015. En Rosario, Sergio Massa prometió usar las Fuerzas Armadas contra el narcotráfico. 23 de septiembre.

La Nación. 2015. Promesa de campaña: Daniel Scioli propuso blindar las fronteras con las Fuerzas Armadas contra el narcotráfico. 02 de noviembre.

La Política Online. 2016. Estudio Nacional de Opinión Pública de Hugo Haime \& Asociados. 19 al 25 de julio. Recuperado de: www.lapoliticaonline.com/files/content/99/.../adelanto Nacional julio 2016.pptx [24 de diciembre de 2016]

López, E. 1987. Seguridad nacional y sedición militar. Buenos Aires: Legasa.

López, E. 2007. Argentina: un largo camino hacia el control civil sobre los militares. En López, E. (Ed.). Control civil sobre los militares y política de defensa en Argentina, Brasil, Chile y Uruguay. Buenos Aires: Altamira.

López, E. 2010. La introducción de la Doctrina de la Seguridad Nacional en el Ejército Argentino. En Moreno, O. (Coord.). La construcción de la Nación Argentina. El rol de las fuerzas armadas. Debates históricos en el marco del Bicentenario 1810/2010. Buenos Aires: Ediciones Ministerio de Defensa.

López, E., Sain, M. (Comp.). 2004. Nuevas Amenazas. Dimensiones y perspectivas. Dilemas y desafíos para la Argentina y el Brasil. Bernal: Universidad Nacional de Quilmes.

Ministerio de Defensa de la República Argentina. 2016a. El ministro Martínez se reunió con la subsecretaria de los Estados Unidos Rose Gottemoeller. Informe de prensa $N^{\circ}$ 120/16. 15 de abril. Recuperado de: http://www.mindef.gov.ar/plantillaNoticia.php? notId=222 [21 de julio de 2016]. 
Ministerio de Defensa de la República Argentina. 2016b. El ministro Martínez se reunió con el jefe del Ejército de Rusia. Informe de prensa $N^{\circ}$ 202/16. 07 de julio. Recuperado de: http://www.mindef.gov.ar/plantillaNoticia.php?notId=222 [21 de julio de 2016].

Montenegro, G. 2008. La misión de las Fuerzas Armadas en la Argentina actual. Revista de la Defensa, 2, 9-19, ago.

Noticias de América Latina y el Caribe (Nodal). 2016. Peña Nieto afirma en la ONU que fracasó el prohibicionismo y la 'guerra contra las drogas'. 20 de abril.

Olson, J., Haugaard, L., Isacson, A. 2007. Below the Radar: U.S. Military Programs with Latin America, 1997- 2007. Publicación conjunta entre el Centro para la Política Internacional, el Fondo de Educación del Grupo de Trabajo sobre América Latina y la Oficina en Washington para Asuntos Latinoamericanos. Washington: WOLA. Recuperado de: http://www.wola.org/sites/default/files/downloadable/Regional\%20Security/past/below the radar final 0307.pdf [21 de julio de 2016].

Pion-Berlin, D. 2008. Militares y democracia en el nuevo siglo. Cuatro descubrimientos inesperados y una conclusión sorprendente. Nueva Sociedad, 213, 50-63.

Sain, M. 2001. Las 'nuevas amenazas' y las Fuerzas Armadas en la Argentina de los '90. Ponencia presentada en la sesión Democracia, militares y nuevas amenazas: Argentina y Brasil en el XXIII Congreso Internacional organizado por Latin American Studies Association, 6-8 septiembre, Washington . Recuperado de: http://lasa.international.pitt.edu/Lasa2001/SainMarcelo.pdf [21 de julio de 2016].

Sain, M. 2013. Dilemas y desafíos en el control del narcotráfico. Página 12. 16 de noviembre.

Sain, M. 2016 La fábula. Le Monde Diplomatique. Febrero.

Salazar, R. 2009. Policialización del ejército y tolerancia cero para los movimientos populares en América Latina. Frónesis, 16 (2), 274-290, ago.

Salvi, V. 2013. Ejército y Nación. Un estudio sobre las estrategias de inscripción de lo/las oficiales del Ejército Argentino en la comunidad nacional. Sociohistórica, 32. Recuperado de: http://www.sociohistorica.fahce.unlp.edu.ar/article/view/

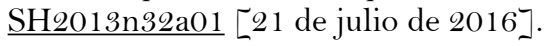

Scheetz, T. 2011. Teoría de la gestión económica de las Fuerzas Armadas. Documento de Trabajo $N^{\circ}$ 7, Serie Documentos de Trabajo. Buenos Aires: Escuela de Defensa Nacional. 
Télam. 2016. Día del Ejército: Macri pidió que las FFAA se adapten a las demandas del siglo XXI. 30 de mayo.

Tello, Á., Spota, J. 2015. Discusiones en torno al rol asignado a la Inteligencia Militar en la Argentina contemporánea. Revista de Relaciones Internacionales, Estrategia y Seguridad, 10 (2), 21-41.

Tokatlian, J. G. 2014. Drogas: lugares comunes que oscurecen la realidad. Clarín. 24 de febrero.

Tres Líneas. 2016. El FPV y la izquierda coincidieron que el decreto de Macri equivale a 'instaurar la pena de muerte', prohibida por la Constitución. 21 de enero.

Ugarte, J. M. 2009. Derribar la Ley de Derribo. Revista de la Defensa, 3, 64-81, ago.

Valero, D. 2016. Santos le propone al mundo un cambio en la lucha contra las drogas. El Tiempo. 21 de abril.

Verbitsky, H. 2011. Posse Comitatus. Página 12. 13 de febrero.

Verbitsky, H. 2012. Las viejas nuevas amenazas. Página 12. 9 de septiembre.

Verbitsky, H. 2016. La transparencia del sigilo. Página 12. 27 de marzo.

Withers, G., Santos, L., Isacson, A. 2010. Preach what you practice: The separation of military and police roles in the Americas. Washington Office on Latin America (WOLA). Recuperado de: http://www.wola.org/sites/default/files/ downloadable/Regional\%20Security/2010/preachwhatyoupractice.pdf $[21$ de julio de 2016]

\section{NORMATIVA}

Ley n ${ }^{\circ} 23554$ de Defensa Nacional.

Ley $n^{\circ} 24059$ de Seguridad Interior.

Ley $n^{\circ} 25520$ de Inteligencia Nacional.

Decreto $n^{\circ}$ 727/2006. Reglamentación de la Ley de Defensa Nacional.

Decreto $n^{\circ}$ 1691/2006. Directiva sobre Organización y Funcionamiento de las Fuerzas Armadas.

Decreto nº 228/2016. Declárase la Emergencia de Seguridad Pública. 


\section{NOTAS}

1. Cedo los derechos de autor a la Asociación Brasileña de Estudios de Defensa, la que podrá incluir el trabajo a ser publicado en bases de datos públicas o privadas, tanto del Brasil como de otros países. Asimismo, soy el único responsable del contenido del texto, el que no contiene nada que pueda ser considerado ilegal o difamatorio de terceras personas.

2. Según un estudio nacional de opinión pública desarrollado en julio de 2016 por la consultora Hugo Haime \& Asociados, los tres principales "problemas del país" son la desocupación, el aumento de precios (inflación) y la corrupción. Ver La Política Online (2016).

3. La misión principal de las Fuerzas Armadas es "conjurar y repeler toda agresión externa militar estatal, a fin de garantizar y salvaguardar de modo permanente los intereses vitales de la Nación, cuales son los de su soberanía, independencia y autodeterminación, su integridad territorial y la vida y libertad de sus habitantes (Decreto $n^{\circ}$ 1691/2006)."

4. La Ley de Defensa Nacional fue sancionada en 1988 durante el gobierno de Raúl Alfonsín; la de Seguridad Interior en 1992 durante la presidencia de Carlos Menem; y la de Inteligencia Nacional en 2001 bajo la presidencia de Fernando de la Rúa. En 2006, luego de 18 años de postergación, el gobierno de Néstor Kirchner reglamentó la Ley de Defensa Nacional (Decreto $n^{\circ}$ 727/2006).

5. Dentro del mismo espacio político se destacaban miradas críticas respecto de esta búsqueda de asignar a los militares un rol en la lucha contra el narcotráfico. Entre ellas, la de la ex ministra de Defensa y de Seguridad Nilda Garré. Ver Garré (2015).

6. Se trata de los siguientes casos: a) operaciones de apoyo logístico, por explícita disposición del Ministro de Defensa, previo requerimiento del Comité de Crisis; b) operaciones destinadas a la preservación de la Fuerza Armada y al restablecimiento del orden dentro de la jurisdicción militar, en caso de atentado en tiempo de paz a dicha jurisdicción; y c) operaciones de empleo de elementos de combate de las Fuerzas Armadas, por disposición del Presidente de la Nación y previa declaración del estado de sitio.

7. Estos "desafíos multifacéticos" remiten a la doctrina de las "nuevas amenazas”, impulsada por los Estados Unidos desde mediados de la década de 1990.

8. Estos académicos han sido convocados para cumplir funciones en el Ministerio de Defensa: el primero como Secretario de Estrategia y Asuntos Militares (viceministro) y el segundo como Director de la Escuela de Defensa Nacional. 
9. Se conoce como "Informe Rattenbach" al documento elaborado por la Comisión de Análisis y Evaluación de las Responsabilidades Políticas y Estratégico Militares en el Conflicto del Atlántico Sur, creada bajo el gobierno de facto de Reynaldo Bignone (1982-83) con el fin de analizar y evaluar el desempeño de las fuerzas armadas argentinas durante la Guerra de Malvinas. En 2012 la presidenta Cristina Fernández estableció - mediante la firma del Decreto nº 200/2012 - el levantamiento del secreto que había establecido la última dictadura militar sobre el Informe.

10. Resulta significativa la línea de investigación que viene desarrollando David Pion-Berlin en relación con las misiones no militares asignadas a las Fuerzas Armadas de los países latinoamericanos. Al respecto, el autor da cuenta de una situación novedosa del siglo XXI: los militares latinoamericanos se han subordinado efectivamente al poder civil, lo que no implicó, sin embargo, su "retiro a los cuarteles". En las últimas dos décadas, los gobiernos de la región han recurrido crecientemente a las estructuras castrenses para la lucha contra el narcotráfico y la delincuencia, y para la asistencia en programas sociales y de desarrollo. Desde la perspectiva de Pion-Berlin ello no ha puesto ni pondrá en peligro - al menos en el corto plazo - la estabilidad democrática. En otras palabras, la era de los golpes de Estado militares habría concluido, aunque no la de la intervención militar en los asuntos domésticos $(2008,53)$.

11. Empleamos el término revival con el objeto de dar cuenta de una discusión que no es nueva, sino que recurrentemente reaparece en la agenda pública de los temas castrenses. Ver Sain (2001) y Verbitsky (2012).

12. Una interesante perspectiva económica sobre este asunto es la de Thomas Scheetz: "Las misiones asignadas a las FFAA deben estar directamente relacionadas a su propio entrenamiento especializado y a su ventaja comparativa, es decir, el uso máximo de la fuerza coercitiva estatal $[\ldots]$ los militares como grupo resultan más caros de entrenar y equipar que, por ejemplo, la policía [...] Emplear a las FFAA como policías o maestros resulta muy caro e ineficiente" (2011, 9-10).

13. Así surge de los discursos pronunciados por los presidentes Juan Manuel Santos (Colombia) y Enrique Peña Nieto (México) en la Sesión Extraordinaria de la Asamblea General de las Naciones Unidas sobre el tema "Drogas". Ver Valero (2016) y Nodal (2016).

14. Referir a la desprofesionalización castrense exhibida en Malvinas no significa desconocer el adecuado desempeño de muchos integrantes de las Fuerzas Armadas argentinas. Como describe el "Informe Rattenbach": "Existen numerosos actos de valor extraordinario, pro- 
ducidos en todas las FFAA (Fuerzas Armadas) y FFSS (Fuerzas de Seguridad) en el teatro de operaciones, por quienes, sirviendo a su deber, acreditaron la vigencia de nuestras mejores tradiciones castrenses" (Comisión de Análisis y Evaluación de las Responsabilidades Políticas y Estratégico Militares en el Conflicto del Atlántico Sur 2012 [1982], 289).

15. El Decreto $\mathrm{n}^{\mathrm{o}}$ 1691/2006 establece como misiones subsidiarias a la participación de las Fuerzas Armadas: a) en el marco de las operaciones multilaterales de Naciones Unidas; b) en operaciones de seguridad interior previstas por la Ley de Seguridad Interior $n^{\circ} 24.059$; ) en operaciones de apoyo a la comunidad nacional o de países amigos; y d) en la construcción de un Sistema de Defensa Subregional.

16. Al desarrollar sus "criterios para aceptar roles de 'uso dual' para las Fuerza Armadas”, Thomas Scheetz considera que el involucramiento de las fuerzas armadas en seguridad interior podría considerarse únicamente para el caso de que las fuerzas opuestas emplearan sistemas de armas mayores y tácticas militares (Scheetz 2011, 80).

17. Según Marcelo Sain: "El narcotráfico se ha transformado en dos sentidos. Por un lado, se produjo una expansión de los mercados internos de drogas ilegales [...] Por otro lado, se intensificó y diversificó el tráfico internacional de cocaína [...] El principal problema del narcotráfico en Argentina es la estructuración de mercados ilegales en grandes ciudades regenteados por grupos criminales con protección y regulación policial $[\ldots]$ la política argentina se ha cuadrado ante las orientaciones de la DEA. Se ha naturalizado que una estrategia exitosa en el control del narcotráfico sólo se consigue mediante una alianza con la agencia” (Sain 2016). 


\section{RESUMEN}

La misión principal de las Fuerzas Armadas se encuentra nuevamente en discusión. A diferencia de la década de 1980, el debate actual no se vincula con la subordinación de los militares al poder constitucional. Hoy los militares argentinos están plenamente consustanciados con el régimen democrático. Sin embargo, el ordenamiento jurídico-normativo del sistema de Defensa se encuentra en una encrucijada. Sectores políticos y académicos plantean la necesidad de revisiones doctrinarias tendientes a facilitar la intervención de los militares en la lucha contra las "nuevas amenazas", en primer lugar el narcotráfico y el terrorismo. Este artículo plantea un conjunto de argumentos político-técnicos contrarios a dicha postura.

Palabras-clave: Misión Principal; Guerra Interestatal; Nuevas Amenazas; Fuerzas Armadas.

\section{ABSTRACT}

The primary mission of the Armed Forces is again under debate in Argentina. Current discussion is not related to the main topic of the 1980s, which was the military subordination to the civil authorities after decades of political involvement. Nowadays, the military are completely subordinated to the democratic institutions. However, the basic legal framework of the National Defense System is at a crossroads. Certain political and academic sectors highlight the need to alter the current normative framework in order to enable the military to play a role against the new global threats such as drug trafficking and terrorism. This article sets out a number of political and technical reasons against this position.

Keywords: Primary Mission; Interstate War; New Threats; Armed Forces. 